
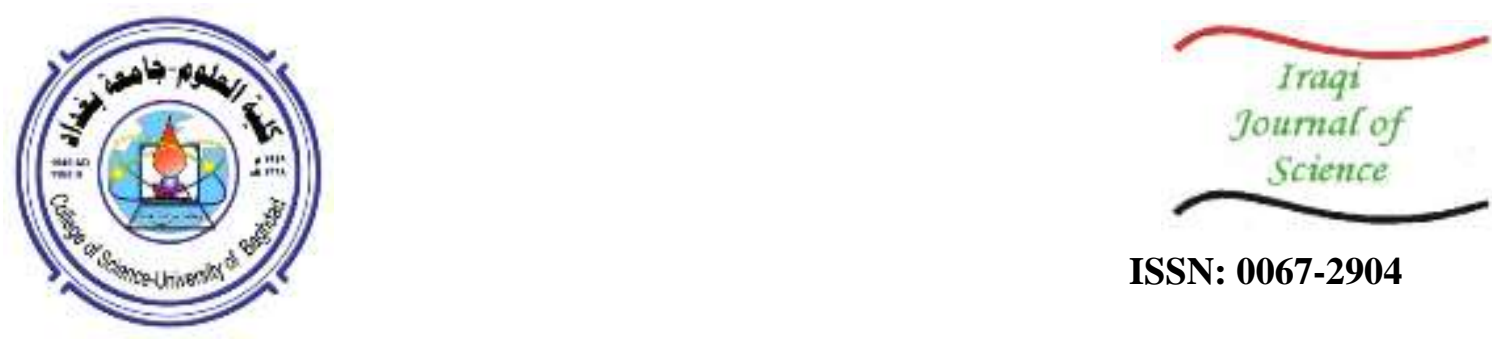

ISSN: 0067-2904

\title{
Modal Analysis of Vibration of Euler-Bernoulli Beam Subjected to Concentrated Moving Load
}

\author{
Usman M. A, Makinde T. A., Daniel D. O. \\ Department of Mathematical Sciences, Olabisi Onabanjo University, Ago-Iwoye, Nigeria
}

Received: 6/9/2019

Accepted: 17/12/2019

\begin{abstract}
This paper investigates the modal analysis of vibration of Euler-Bernoulli beam subjected to concentrated load. The governing partial differential equation was analysed to determine the behaviour of the system under consideration. The series solution and numerical methods were used to solve the governing partial differential equation. The results revealed that the amplitude increases as the length of the beam increases. It was also found that the response amplitude increases as the foundation increases at fixed length of the beam.
\end{abstract}

Keywords: Euler-Bernoulli Beam, Concentrated Moving Load, Vibration, Amplitude, Moving Load.

\section{Introduction}

Beams are fundamental models for the structural elements of many engineering applications and they were extensively studied. Every structure which has some mass and elasticity is said to be vibrate [1]. Research on vibrations of beams was conducted by many authors for a long period of time. So far, many authors introduced different methods to find the free vibration behaviour of shear flexible beams. Abrate [2] analysed the free vibration of non-uniform beams with general shape and arbitrary boundary conditions. Simple formulas were presented for predicting the fundamental natural frequency of non-uniform beams with various end support conditions. An earlier work [3] studied free vibrations of tapered beams with general boundary conditions. This method involves finding the ordinary differential governing equation of beams which can be solved by numerical methods. The natural frequencies are calculated by combining the Runge-Kutta method and the determinant search method. Studies were also conducted on the dynamic behaviour of beams with linearly varying crosssections, where the equation of motion was solved using an analytical method to the initial boundary vale problem described by the governing equation $[4,5,6,7]$.

i. The aim of this research is to determine the dynamic response of a pipeline-supported beam on an elastic foundation resting on a Winkler foundation, in order to obtain the dynamic responses such as deflection and bending moments [8-11]. The objectives of this study are to present the analysis of Euler-Bernoulli beam, subjected to partially distributed moving load and to find the analytical solution of the governing partial differential equation of the beam [12-15]. We also aimed at determining the displacement of the beam as a function of time and distance $\beta(x, t)$, subject to initial and boundary conditions of the system [16-20].

\section{Mathematical Formulation}

Consider a non-prismatic Euler-Bernoulli beam of a length L, resting on a Winkler foundation and transverse by uniform partially distributed moving load [21-24]. 


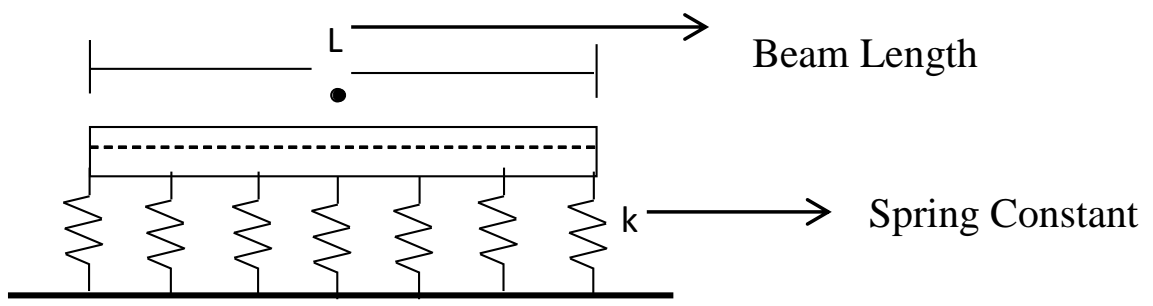

Figure 1-Geometry of the problem.

The resulting vibration behaviour of this system is described by the following partial differential equations.

A forced vibration model exists in which there is a moving load in the form of fluid on the beam. The assumed solution is in the form of Fourier series solution and the resulting vibration behaviour of the beam is described by the fourth order partial differential equation below $[25,26,27,28,29,30]$ :

in the case of moving force,

$$
E I \frac{\partial^{4} \beta}{\partial x^{4}}+K(x) \beta+\rho A(x)\left[\frac{\partial^{2} \beta}{\partial t^{2}}\right]=F(x, t)
$$

where,

$$
F(x, t)=P \delta(x-v t)
$$

where $\mathrm{E}$ is Young's modulus, $\mathrm{I}$ is the area of the moment of inertia about the neutral axis $\left(\mathrm{m}^{4}\right), \rho$ is the mass density $\left(\mathrm{kg} / \mathrm{m}^{3}\right), \beta$ is the deflection $(\mathrm{m})$ or the transverse displacement of a segment of the beam along $x, x$ is the horizontal space coordinate measure along the length of the beam, $A$ is the cross sectional area of the beam $\left(\mathrm{m}^{2}\right), t$ is any particular instant of time in seconds, $K$ is spring constant per unit length, $\delta$ is Dirac delta function, and $P \delta(x-v t)$ is the applied moving force per unit mass.

\subsection{ASSUMPTIONS}

Assumptions taken into consideration are:

\section{i. Structural Assumption:}

(a) Initially straight beam

(b) Linear elastic material

(c) Small structural deformation

ii. Shear deformation and rotary inertial effect are neglected (Euler-Bernoulli beam). Hence, the length ratio of the beam is small.

iii. Weight of the moving load is larger than the mass of the beam, so we will consider only gravitational effect of load.

iv. Load is moving at a constant speed.

\subsection{Method of Solution}

The deflection mode of the continuous beam with simply-supported or position-dependent boundary conditions can be derived from the equation of the beam. The deflection modes and the natural frequencies of the beam can be expressed as:

$$
Y_{n}(x)=\sin \frac{n \pi x}{l} \text {, and } \beta_{n}=\beta(x, t)=\left(\frac{n \pi}{l}\right)^{2} \sqrt{\frac{E I}{\rho A}}, \text { where } n=1,2,3 \ldots N
$$

where $Y_{n}(x)$ is the deflection mode, $w_{n}$ is the corresponding natural frequency, and $l$ is the beam length.

\subsection{MODAL ANALYTICAL SOLUTION}

Assume a series solution of equation_(1.0) in the form of a series

$$
\beta(x, t)=\sum_{n=1}^{N} Y_{n}(x) q_{n}(t) \quad n=1,2,3, \ldots N
$$

where $Y_{n}(x)$ is the eigen function of the beam, $q_{n}(t)$ is a function of time which must be found, and $n$ is the number of contributed modes. 


$$
\begin{gathered}
Y_{n}(x)=\sin \frac{n \pi x}{l} \\
q_{n}(t)=\frac{2 P}{\rho A l \omega_{n}^{2}} \times \frac{1}{2}\left(\sin \omega_{n} \cdot t-\omega_{n} \cdot t \cos \omega_{n} \cdot t\right) \\
\omega_{n}^{2}=\frac{n^{4} \pi^{4} E I}{\rho A l^{4}} \\
\omega_{n}=\left(\frac{n \pi}{l}\right)^{2} \sqrt{\frac{E I}{\rho A}} \\
q_{n}(t)=2 P \div \rho A l \times\left(\frac{n \pi}{l}\right)^{4} \frac{E I}{\rho A} \times \frac{1}{2}\left(\sin \left(\frac{n \pi}{l}\right)^{2} \sqrt{\frac{E I}{\rho A}} \cdot t-\left(\frac{n \pi}{l}\right)^{2} \sqrt{\frac{E I}{\rho A}} \times \cos \left(\frac{n \pi}{l}\right)^{2} \sqrt{\frac{E I}{\rho A}} \cdot t\right) \\
q_{n}(t)=\frac{P l^{3}}{(n \pi)^{4} E I}\left(\sin \left(\frac{n \pi}{l}\right)^{2} \sqrt{\frac{E I}{\rho A}} \cdot t-\left(\frac{n \pi}{l}\right)^{2} \sqrt{\frac{E I}{\rho A}} \times \cos \left(\frac{n \pi}{l}\right)^{2} \sqrt{\frac{E I}{\rho A}} \cdot t\right) \\
\left.\times \cos \left(\frac{n \pi}{l}\right)^{2} \sqrt{\frac{E I}{\rho A}} \cdot t\right) \times \sin \frac{n \pi v t}{l} \\
\quad(1.4)
\end{gathered}
$$

Note that $\quad\left[\sin \frac{n \pi x}{l}=\sin \frac{n \pi v t}{l}\right]_{x=v t}$

$$
E I \frac{\partial^{4} \beta}{\partial x^{4}}+K(x) \beta+\rho A(x)\left(\frac{\partial^{2} \beta}{\partial t^{2}}\right)=F(x, t)
$$

Where $F(x, t)=P \delta(x-v t)$ becomes

$$
\mathrm{EI} \frac{\partial^{4} \beta}{\partial x^{4}}+K(x) \beta+\rho A(x)\left(\frac{\partial^{2} \beta}{\partial t^{2}}\right)=P \delta(x-v t)
$$

We substitute Eq.(1.2) together with Eq.(1.3) into Eq. (1.11) as follows;

$$
\begin{gathered}
E I \sum_{n=1}^{N} \sin \left[\frac{n \pi x}{l}\right]^{4} q_{n}(t)+K \sum_{n=1}^{N} \sin \left[\frac{n \pi x}{l}\right] q_{n}(t)+\rho A \sum_{n=1}^{N} \sin \left[\frac{n \pi x}{l}\right] \ddot{q}_{n}(t) \\
=P \sum_{n=1}^{N} \sin \left[\frac{n \pi x}{l}\right](v t)
\end{gathered}
$$

Using simplified subscript for differentiation in Eq.(1.12) gives

$$
E I\left[\frac{n \pi}{l}\right]^{4} \sum_{n=1}^{N} Y_{n}(x) q_{n}(t)+K \sum_{n=1}^{N} Y_{n}(x) q_{n}(t)+\rho A \sum_{n=1}^{N} Y_{n}(x) \ddot{q}_{n}(t)=P \sum_{n=1}^{N} Y_{n}(v t)
$$

Multiplying both sides of equation (1.13) by $Y_{p}(x)$ results in

$$
\begin{gathered}
E I\left[\frac{n \pi}{l}\right]^{4} \sum_{n=1}^{N} Y_{n}(x) q_{n}(t) Y_{p}(x)+K \sum_{n=1}^{N} Y_{n}(x) q_{n}(t) Y_{p}(x)+\rho A \sum_{n=1}^{N} Y_{n}(x) \ddot{q}_{n}(t) Y_{p}(x) \\
=P \sum_{n=1}^{N} Y_{n}(v t) Y_{p}(v t)
\end{gathered}
$$

Integrating along the beam length and employing the orthogonality property among the normal modes gives 


$$
\begin{gathered}
E I\left[\frac{n \pi}{l}\right]^{4} \sum_{n=1}^{N} q_{n}(t) \int_{0}^{l} Y_{n}(x) Y_{p}(x) d x+K \sum_{n=1}^{N} q_{n}(t) \int_{0}^{l} Y_{n}(x) Y_{p}(x) d x+\rho A \sum_{n=1}^{N} \ddot{q}_{n}(t) \int_{0}^{l} Y_{n}(x) Y_{p}(x) d x \\
=P \sum_{n=1}^{N} Y_{n}(v t) Y_{p}(v t) d v t
\end{gathered}
$$

Which gives

$$
\begin{gathered}
E I\left[\frac{n \pi}{l}\right]^{4} \sum_{n=1}^{N} q_{n}(t) \int_{0}^{l}\left[Y_{n}(x)\right]^{2} d x+K \sum_{n=1}^{N} q_{n}(t) \int_{0}^{l}\left[Y_{n}(x)\right]^{2} d x+\rho A \sum_{n=1}^{N} \ddot{q}_{n}(t) \int_{0}^{l}\left[Y_{n}(x)\right]^{2} d x \\
=P \sum_{n=1}^{N} Y_{n}(v t) Y_{p}(v t) d v t
\end{gathered}
$$

The definition of function of orthogonality is applied to give the LHS of equation (1.16). The definition is as follows:

(1) The two non-zero functions $f(x)$ and $g(x)$ are said to be orthogonal on $a \leq x \leq b$ if

$$
\int_{a}^{b} f(x) g(x) d x=0
$$

(2) A set of non-zero function $\left\{f_{i}(x)\right\}$ is said to be mutually orthogonal or orthogonal set on $a \leq x \leq b$ if $f_{i}(x)$ and $f_{j}(x)$ are orthogonal for every $i \neq j$. In other words

$$
\int_{a}^{b} f_{i}(x) f_{j}(x) d x=\left\{\begin{array}{c}
0 \text { if } i \neq j \\
c>0 \text { if } i=j
\end{array}\right.
$$

Note that in the case of $i=j$ for the second definition, we know that we will get a positive value from the integral, because

$$
\int_{a}^{b} f_{i}(x) f_{i}(x) d x=\int_{a}^{b}\left[f_{i}(x)\right]^{2} d x>0
$$

Recall that when we integrate a positive function, we know that the result will be positive as well. Similarly, the non-zero requirement is important because otherwise the integral would be trivially zero regardless of the function.

In the case of equation (1.15) above, $n=p$, which gives equation (1.16). After integration and some rearrangements, equation (1.16) gives

For $n=1,2,3, \ldots, N$.

$$
\frac{E I}{\rho A}\left(\frac{n \pi}{l}\right)^{4} q_{n}(t)+\ddot{q}_{n}(t)+\frac{K}{\rho A} q_{n}(t)=\frac{2 P}{\rho A l} \sum_{n=1}^{N} Y_{n}(v t) Y_{p}(v t)
$$

Since $n=p$, equation (17) becomes

$$
\frac{E I}{\rho A}\left(\frac{n \pi}{l}\right)^{4} q_{n}(t)+\ddot{q}_{n}(t)+\frac{K}{\rho A} q_{n}(t)=\frac{2 P}{\rho A l} \sum_{n=1}^{N}\left(Y_{n}(v t)\right)^{2}
$$

Equation (1.21) is an ordinary differential equation, and a numerical procedure shall be employed to solve it. It can be also simplified further by substituting the unknown functions in the equation. The unknown functions are:

From equation (1.3) and (1.8), $Y_{n}(x)=Y_{n}(v t)=\sin \frac{n \pi x}{l}$ and

$$
q_{n}(t), \ddot{q}_{n}(t) \text { and } Y_{n}(v t)
$$

$$
q_{n}(t)=\frac{P l^{3}}{(n \pi)^{4} E I}\left(\sin \left(\frac{n \pi}{l}\right)^{2} \sqrt{\frac{E I}{\rho A}} \cdot t-\left(\frac{n \pi}{l}\right)^{2} \sqrt{\frac{E I}{\rho A}} \times \cos \left(\frac{n \pi}{l}\right)^{2} \sqrt{\frac{E I}{\rho A}} \cdot t\right)
$$

Substituting $q_{n}(t)$ and $Y_{n}(v t)$ into equation (1.21), gives 


$$
\begin{gathered}
\frac{E I}{\rho A}\left(\frac{n \pi}{l}\right)^{4} \frac{P l^{3}}{(n \pi)^{4} E I}\left(\sin \left(\frac{n \pi}{l}\right)^{2} \sqrt{\frac{E I}{\rho A}} \cdot t-\left(\frac{n \pi}{l}\right)^{2} \sqrt{\frac{E I}{\rho A}} \times \cos \left(\frac{n \pi}{l}\right)^{2} \sqrt{\frac{E I}{\rho A}} \cdot t\right)+\ddot{q}_{n}(t) \\
+\frac{K}{\rho A} \frac{P l^{3}}{(n \pi)^{4} E I}\left(\sin \left(\frac{n \pi}{l}\right)^{2} \sqrt{\frac{E I}{\rho A}} \cdot t-\left(\frac{n \pi}{l}\right)^{2} \sqrt{\frac{E I}{\rho A}} \times \cos \left(\frac{n \pi}{l}\right)^{2} \sqrt{\frac{E I}{\rho A}} \cdot t\right) \\
=\frac{2 P}{\rho A l} \sum_{n=1}^{N}\left(\sin \left(\frac{n \pi x}{l}\right)\right)^{2} \\
\ddot{q}_{n}(t)+\omega_{n}^{2} q_{n}(t)=\left(\frac{2 P}{\rho A l}\right) \sin \bar{\omega}_{n} \cdot t \\
\ddot{q}_{n}(t)=\left(\frac{2 P}{\rho A l}\right) \sin \bar{\omega}_{n} \cdot t-\omega_{n}^{2} q_{n}(t)
\end{gathered}
$$

where

$$
\omega_{n}^{2}=\frac{n^{4} \pi^{4} E I}{\rho A l^{4}}, \bar{\omega}_{n}=\frac{n \pi v}{l}
$$

and

$$
q_{n}(t)=\frac{P l^{3}}{(n \pi)^{4} E I}\left(\sin \left(\frac{n \pi}{l}\right)^{2} \sqrt{\frac{E I}{\rho A}} \cdot t-\left(\frac{n \pi}{l}\right)^{2} \sqrt{\frac{E I}{\rho A}} \times \cos \left(\frac{n \pi}{l}\right)^{2} \sqrt{\frac{E I}{\rho A}} \cdot t\right)
$$

Substituting equation (1.26) and (1.27) into equation (1.25) gives

$$
\begin{aligned}
\ddot{q}_{n}(t)=\left(\frac{2 P}{\rho A l}\right) & \sin \frac{n \pi v}{l} \cdot t \\
& -\frac{n^{4} \pi^{4} E I}{\rho A l^{4}} \cdot \frac{P l^{3}}{(n \pi)^{4} E I}\left(\sin \left(\frac{n \pi}{l}\right)^{2} \sqrt{\frac{E I}{\rho A}} \cdot t-\left(\frac{n \pi}{l}\right)^{2} \sqrt{\frac{E I}{\rho A}} \times \cos \left(\frac{n \pi}{l}\right)^{2} \sqrt{\frac{E I}{\rho A}} \cdot t\right)(1
\end{aligned}
$$

Simplifying equation (1.28) gives

$$
\begin{aligned}
& \ddot{q}_{n}(t)=\left(\frac{2 P}{\rho A l}\right) \sin \frac{n \pi v}{l} \cdot t-\frac{P}{\rho A l}\left(\sin \left(\frac{n \pi}{l}\right)^{2} \sqrt{\frac{E I}{\rho A}} \cdot t-\left(\frac{n \pi}{l}\right)^{2} \sqrt{\frac{E I}{\rho A}} \times \cos \left(\frac{n \pi}{l}\right)^{2} \sqrt{\frac{E I}{\rho A}} \cdot t\right) \\
& \ddot{q}_{n}(t)=\frac{P}{\rho A l}\left(2\left(\sin \frac{n \pi v}{l} \cdot t\right)-1\left(\sin \left(\frac{n \pi}{l}\right)^{2} \sqrt{\frac{E I}{\rho A}} \cdot t-\left(\frac{n \pi}{l}\right)^{2} \sqrt{\frac{E I}{\rho A}} \times \cos \left(\frac{n \pi}{l}\right)^{2} \sqrt{\frac{E I}{\rho A}} \cdot t\right)\right)
\end{aligned}
$$

By substituting equation (1.30) into equation (1.23), it becomes

$$
\begin{aligned}
\frac{E I}{\rho A}\left(\frac{n \pi}{l}\right)^{4} \frac{P l^{3}}{(n \pi)^{4} E I}\left(\sin \left(\frac{n \pi}{l}\right)^{2} \sqrt{\frac{E I}{\rho A}} \cdot t-\left(\frac{n \pi}{l}\right)^{2} \sqrt{\frac{E I}{\rho A}} \times \cos \left(\frac{n \pi}{l}\right)^{2} \sqrt{\frac{E I}{\rho A}} \cdot t\right) \\
+\frac{P}{\rho A l}\left(2\left(\sin \frac{n \pi v}{l} \cdot t\right)\right. \\
\left.-1\left(\sin \left(\frac{n \pi}{l}\right)^{2} \sqrt{\frac{E I}{\rho A}} \cdot t-\left(\frac{n \pi}{l}\right)^{2} \sqrt{\frac{E I}{\rho A}} \times \cos \left(\frac{n \pi}{l}\right)^{2} \sqrt{\frac{E I}{\rho A}} \cdot t\right)\right) \\
+\frac{K}{\rho A} \frac{P l^{3}}{(n \pi)^{4} E I}\left(\sin \left(\frac{n \pi}{l}\right)^{2} \sqrt{\frac{E I}{\rho A}} \cdot t-\left(\frac{n \pi}{l}\right)^{2} \sqrt{\frac{E I}{\rho A}} \times \cos \left(\frac{n \pi}{l}\right)^{2} \sqrt{\frac{E I}{\rho A}} \cdot t\right) \\
=\frac{2 P}{\rho A l} \sum_{n=1}^{N}\left(\sin \left(\frac{n \pi x}{l}\right)\right)^{2}
\end{aligned}
$$


Simplifying equation (1.31) gives

$$
\begin{aligned}
\frac{P l^{3}}{\rho A(n \pi)^{4} E I}(( & \left.\left.\sin \left(\frac{n \pi}{l}\right)^{2} \sqrt{\frac{E I}{\rho A}} \cdot t-\left(\frac{n \pi}{l}\right)^{2} \sqrt{\frac{E I}{\rho A}} \times \cos \left(\frac{n \pi}{l}\right)^{2} \sqrt{\frac{E I}{\rho A}} \cdot t\right)\right)\left[E I\left(\frac{n \pi}{l}\right)^{4}+K\right] \\
& +\frac{P}{\rho A l}\left(2\left(\sin \frac{n \pi v}{l} \cdot t\right)\right. \\
& \left.-1\left(\sin \left(\frac{n \pi}{l}\right)^{2} \sqrt{\frac{E I}{\rho A}} \cdot t-\left(\frac{n \pi}{l}\right)^{2} \sqrt{\frac{E I}{\rho A}} \times \cos \left(\frac{n \pi}{l}\right)^{2} \sqrt{\frac{E I}{\rho A}} \cdot t\right)\right) \\
= & \frac{2 P}{\rho A l} \sum_{n=1}^{N}\left(\sin \left(\frac{n \pi x}{l}\right)\right)^{2}
\end{aligned}
$$

Multiply equation (1.32) by $\rho A$, gives

$$
\begin{gathered}
\frac{P l^{3}}{(n \pi)^{4} E I}\left(\left(\sin \left(\frac{n \pi}{l}\right)^{2} \sqrt{\frac{E I}{\rho A}} \cdot t-\left(\frac{n \pi}{l}\right)^{2} \sqrt{\frac{E I}{\rho A}} \times \cos \left(\frac{n \pi}{l}\right)^{2} \sqrt{\frac{E I}{\rho A}} \cdot t\right)\right)\left[E I\left(\frac{n \pi}{l}\right)^{4}+K\right] \\
+\frac{P}{l}\left(2\left(\sin \frac{n \pi v}{l} \cdot t\right)-1\left(\sin \left(\frac{n \pi}{l}\right)^{2} \sqrt{\frac{E I}{\rho A}} \cdot t-\left(\frac{n \pi}{l}\right)^{2} \sqrt{\frac{E I}{\rho A}} \times \cos \left(\frac{n \pi}{l}\right)^{2} \sqrt{\frac{E I}{\rho A}} \cdot t\right)\right) \\
=\frac{2 P}{l} \sum_{n=1}^{N}\left(\sin \left(\frac{n \pi x}{l}\right)\right)^{2}
\end{gathered}
$$

Equation (1.33) above is the simplified form of equation (1.23) above, i.e.,

$$
\frac{E I}{\rho A}\left(\frac{n \pi}{l}\right)^{4} q_{n}(t)+\ddot{q}_{n}(t)+\frac{K}{\rho A} q_{n}(t)=\frac{2 P}{\rho A l} \sum_{n=1}^{N}\left(Y_{n}(v t)\right)^{2}
$$

While, the modal deflection of the beam in equation (1.11) is given as:

$$
w(x, t)=\sum_{n=1}^{N} \frac{P l^{3}}{(n \pi)^{4} E I}\left(\sin \left(\frac{n \pi}{l}\right)^{2} \sqrt{\frac{E I}{\rho A}} \cdot t-\left(\frac{n \pi}{l}\right)^{2} \sqrt{\frac{E I}{\rho A}} \times \cos \left(\frac{n \pi}{l}\right)^{2} \sqrt{\frac{E I}{\rho A}} \cdot t\right) \times \sin \frac{n \pi v t}{l}
$$

\section{Result and Discussion}

The beam was subjected to a moving load; the equation considered a fourth order Partial Differential Equation (PDE). The PDE was transformed to an ordinary differential equation. The equation was solved with an assumed solution in form of series. A software package was used to analyse the solution obtained graphically with the parameters. Graphs are presented in Figures (2-7) below. The dynamic responses, such as deflection and bending moment of the beam, were obtained under different velocities $(v)$, contributed mode $(n)$, and time $(t)$ domain.

From Figure-2, it is observed that the deflection is somehow symmetric at a minimum point. The figure also shows that the maximum dynamic deflection for both upward and downward directions increases and decreases, respectively, with the increase in velocity. The maximum bending moment occurs at $0.1851,0.2637$ and 0.3672 while the minimum bending moment takes place at -0.0991 , 0.1406 and -0.1945 , respectively, for the velocities.

Figure-3 demonstrates the deflection tilted towards the positive side rather than the negative side of the beam, with minimum bending moments of $-0.0661,-0.0938$ and -0.1297 and maximum deflections at $0.1092,0.1470$ and 0.1831 , respectively. The figure further explains that the deflection is wider upward and narrows at the downward part of the beam.

In Figure- $4 \mathrm{a}$ and $4 \mathrm{~b}$, the same parameter were used in each part of the figure; fig.4a was timepartitioned along the different point of the beam while fig. $4 \mathrm{~b}$ was not partitioned. It is observed that 
both graphs pick their maximum deflection towards the end of the beam, with varying minimum bending moment values for different velocities, almost at the same point of the beam.

Figure-5 shows that the minimum bending moment of the deflection is approximately symmetric at a point for all the selected velocities, and the maximum bending moment occurs just at the beginning of the beam, with the successive crest of the maximum velocity being almost the same. The maximum deflection occurs at $1.0505,1.4138$ and 1.7618 while the minimum deflection occurs at -0.7633 , 1.0743 and -1.4637 , respectively, for all the velocities, as shown in the figure.

Figure-6 illustrates that both the deflection and bending moments are more visible as they progress along the beam length, with more deflection noticed at the downward part of the beam. The minimum and maximum deflections for the maximum velocity are 0.58773 and -0.6342 , respectively.

In Figure-7, it is observed that the visible maximum deflection occurs at the upper part of the beam, while the deflection damps almost from the middle to the end of the beam, with maximum and minimum bending moments of 1.4836 and -0.4039 , respectively, for the maximum velocity.

It shows the analysis under the same condition of contributed modes, with different time domains.

Table 1-The parameters used in this study along with their respective values

\begin{tabular}{|c|c|}
\hline Parameters & Value(s) \\
\hline$\rho$ & $25 \mathrm{~kg} / \mathrm{m}^{3}$ \\
\hline$E I$ & $2.1528 \times 10^{5} \mathrm{~N} / \mathrm{m}^{2}$ \\
\hline$P$ & $9.936 \times 10 \mathrm{~N}$ \\
\hline$l$ & $10 \mathrm{~m}$ \\
\hline $\mathrm{A}$ & $2.45 \mathrm{~m}^{2}$ \\
\hline$V$ & $7 \mathrm{~m} / \mathrm{s}, 10 \mathrm{~m} / \mathrm{s}, 14 \mathrm{~m} / \mathrm{s}$ \\
\hline$n(3.1)$ & $1,2,3$ \\
\hline$k(3.2)$ & $1,2,3$ \\
\hline$t$ & $1 \mathrm{~s}, 2 \mathrm{~s}, 3 \mathrm{~s}$ \\
\hline$m$ & $10 \mathrm{~kg}, 15 \mathrm{~kg}, 20 \mathrm{~kg}$ \\
\hline$h$ & $0.5,1.0,1.5$ \\
\hline$c_{1}$ & $0.1,0.2,0.3$ \\
\hline
\end{tabular}

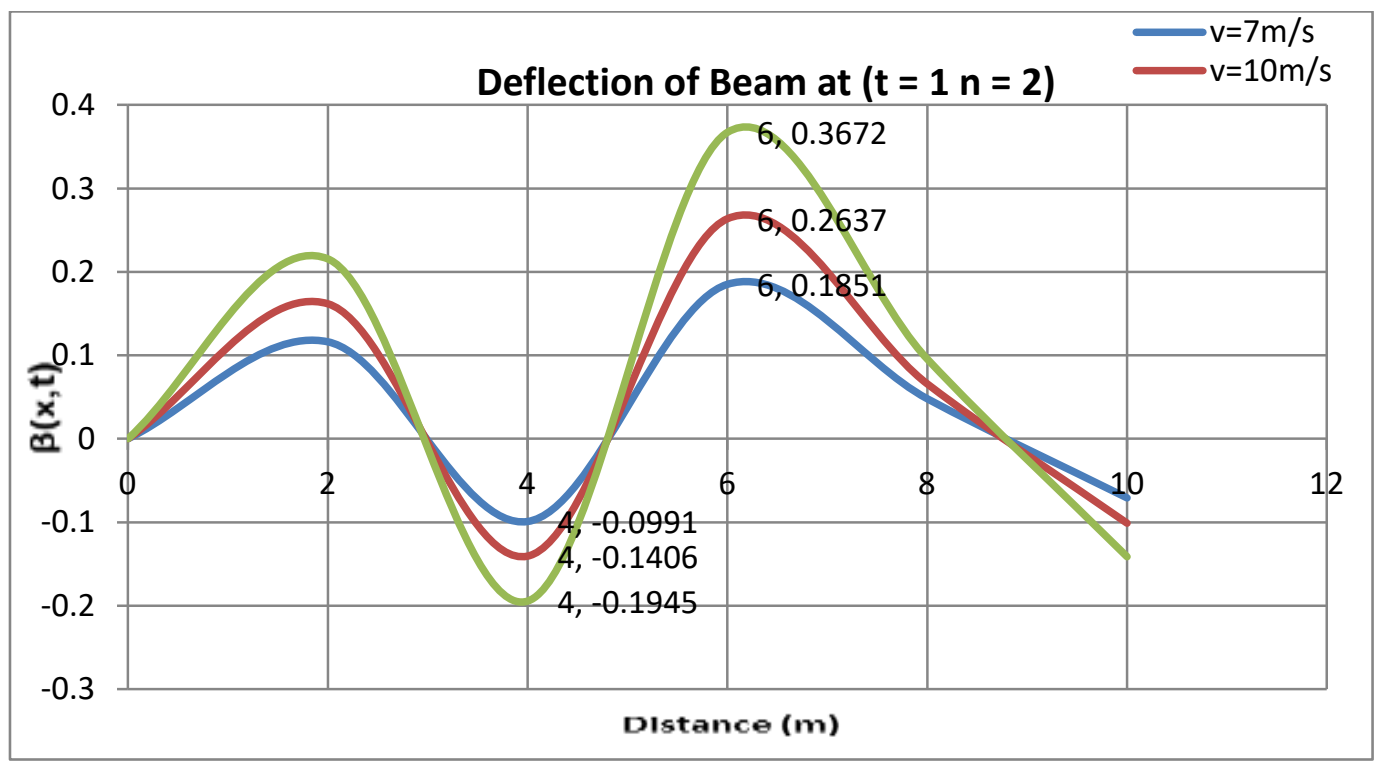

Figure 2-Graph of deflection at $\mathrm{t}=1 \mathrm{~s}(\mathrm{NP})$ and $\mathrm{n}=2$ 


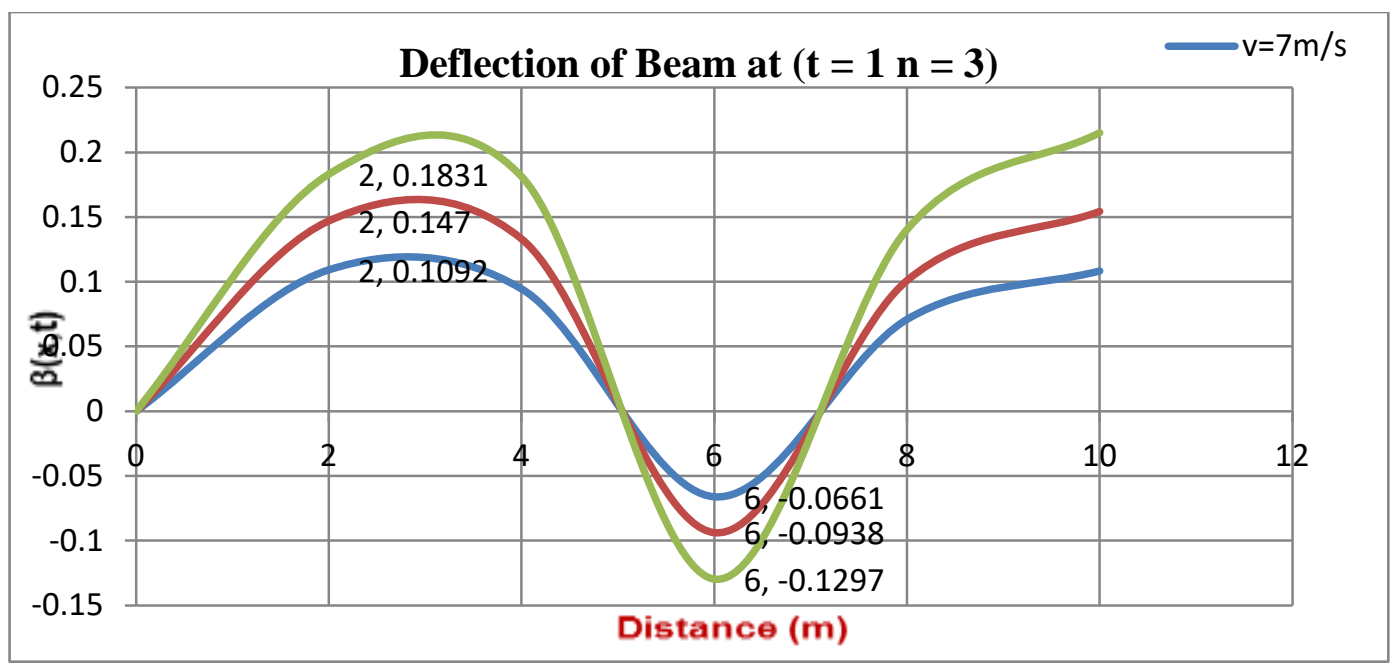

Figure 3-Graph of deflection at $t=1 \mathrm{~s}(\mathrm{NP})$ and $n=3$

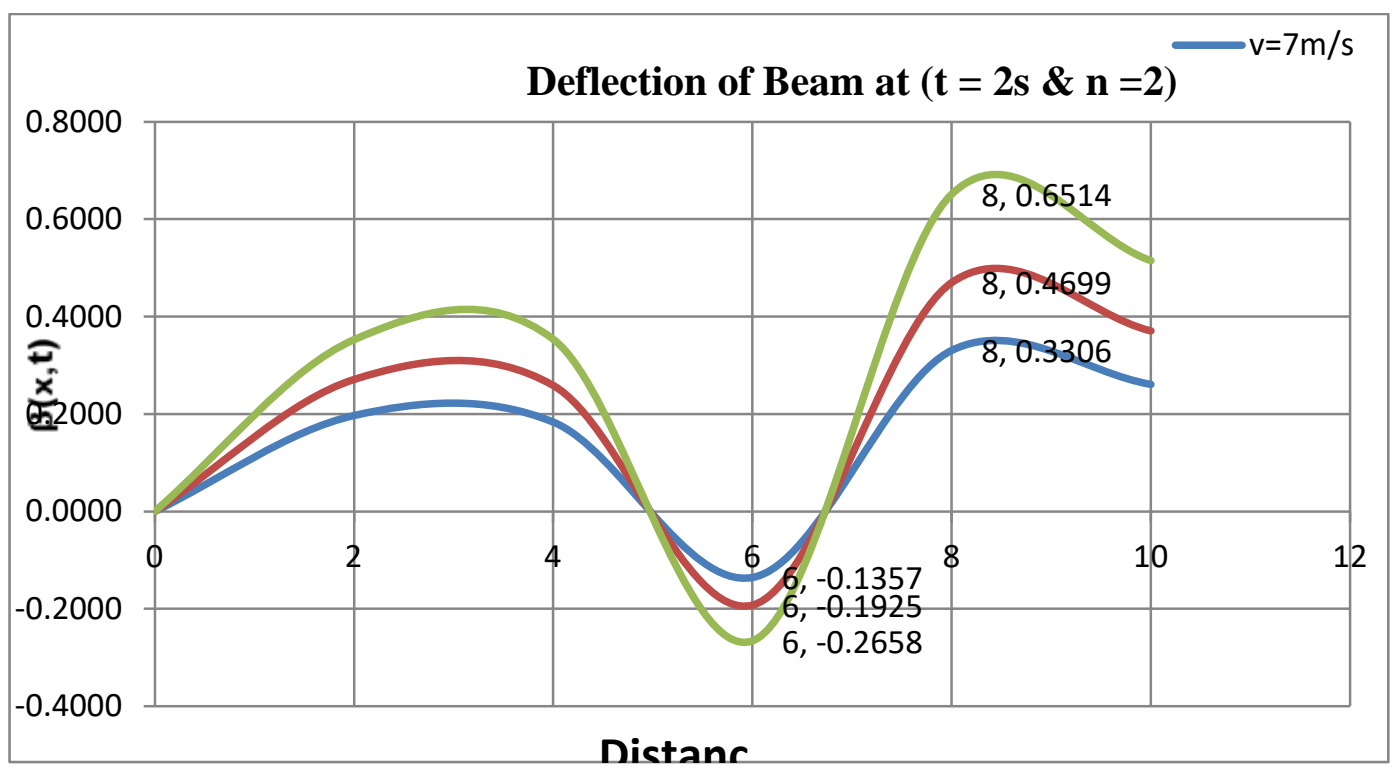

Figure 4a-Graph of deflection at $\mathrm{t}=2 \mathrm{~s}$ (partitioned) and $\mathrm{n}=2$

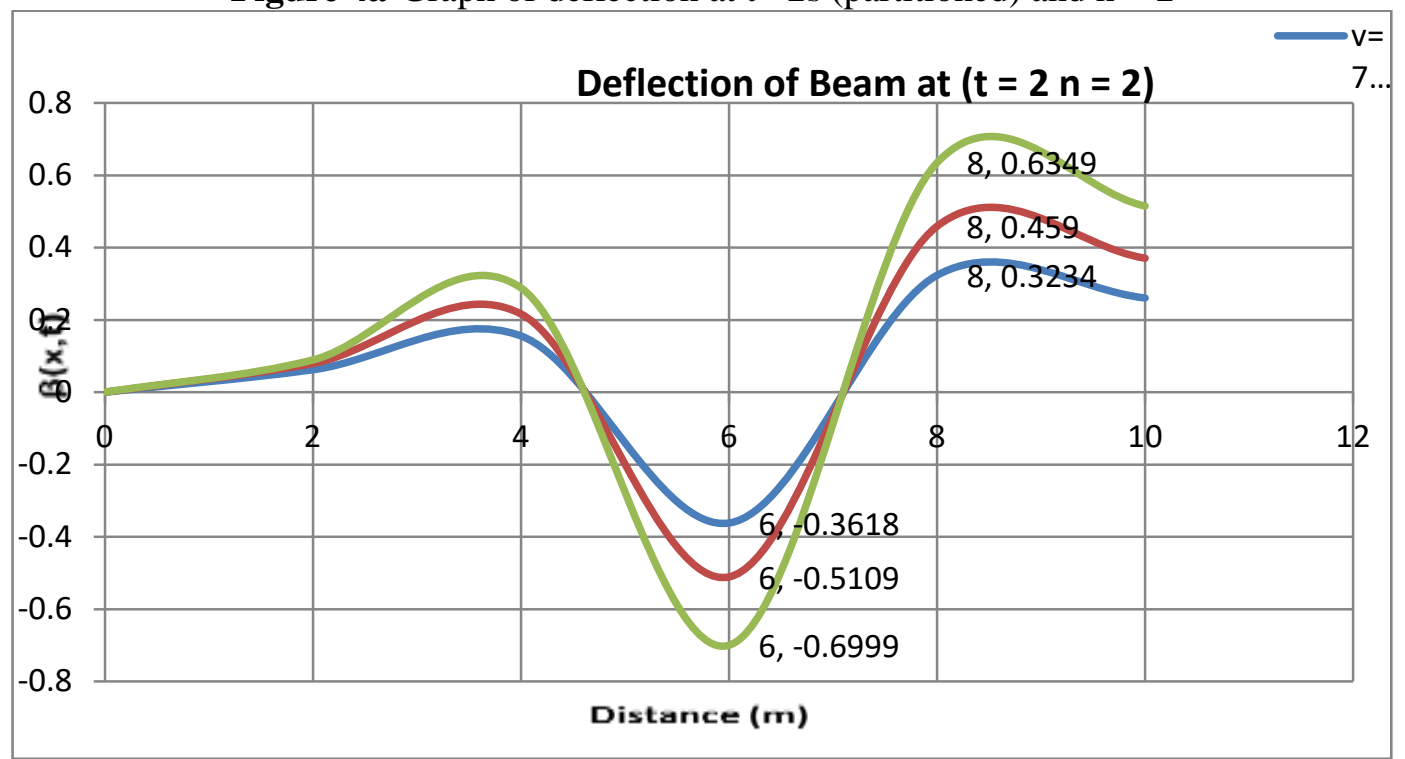

Figure 4b-Graph of deflection at $t=2 s(\mathrm{NP})$ and $n=2$ 


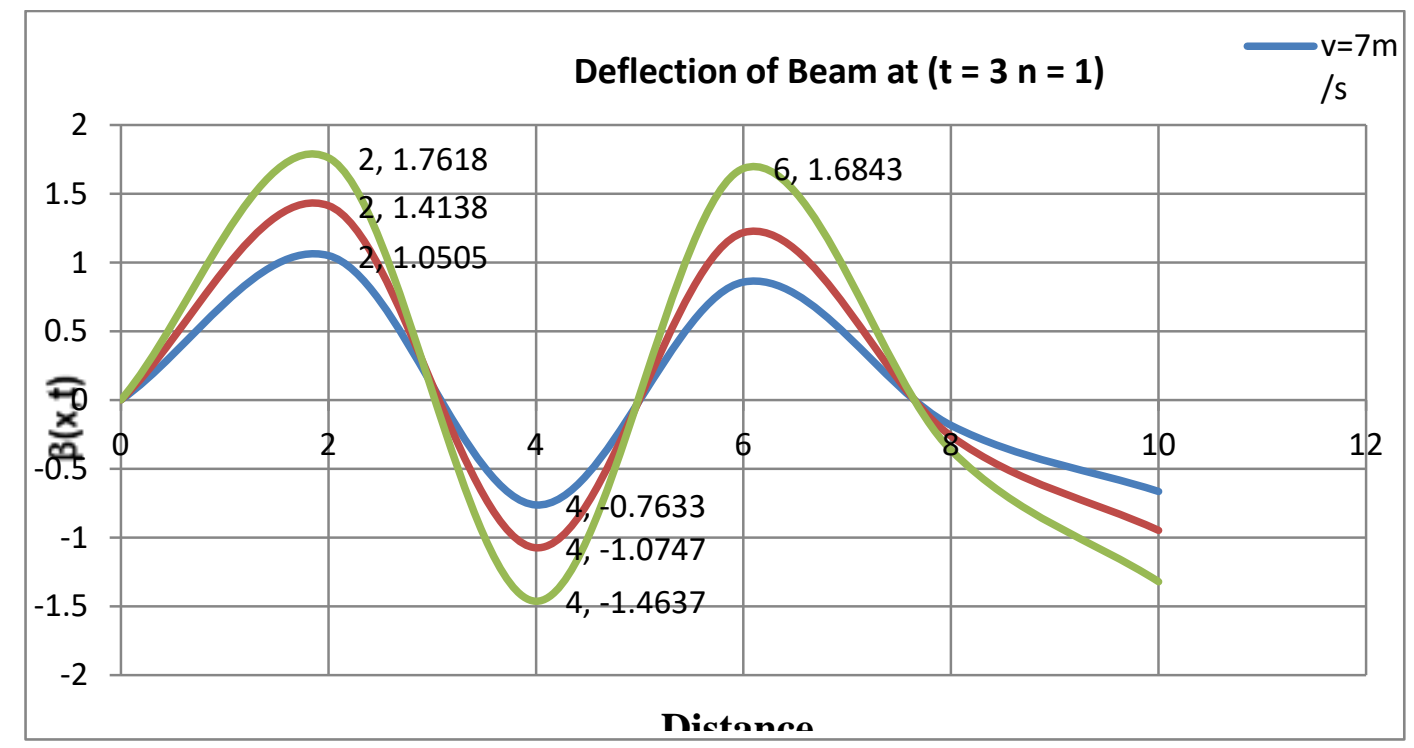

Figure 5-Graph of deflection at $\mathrm{t}=3 \mathrm{~s}(\mathrm{NP})$ and $\mathrm{n}=1$

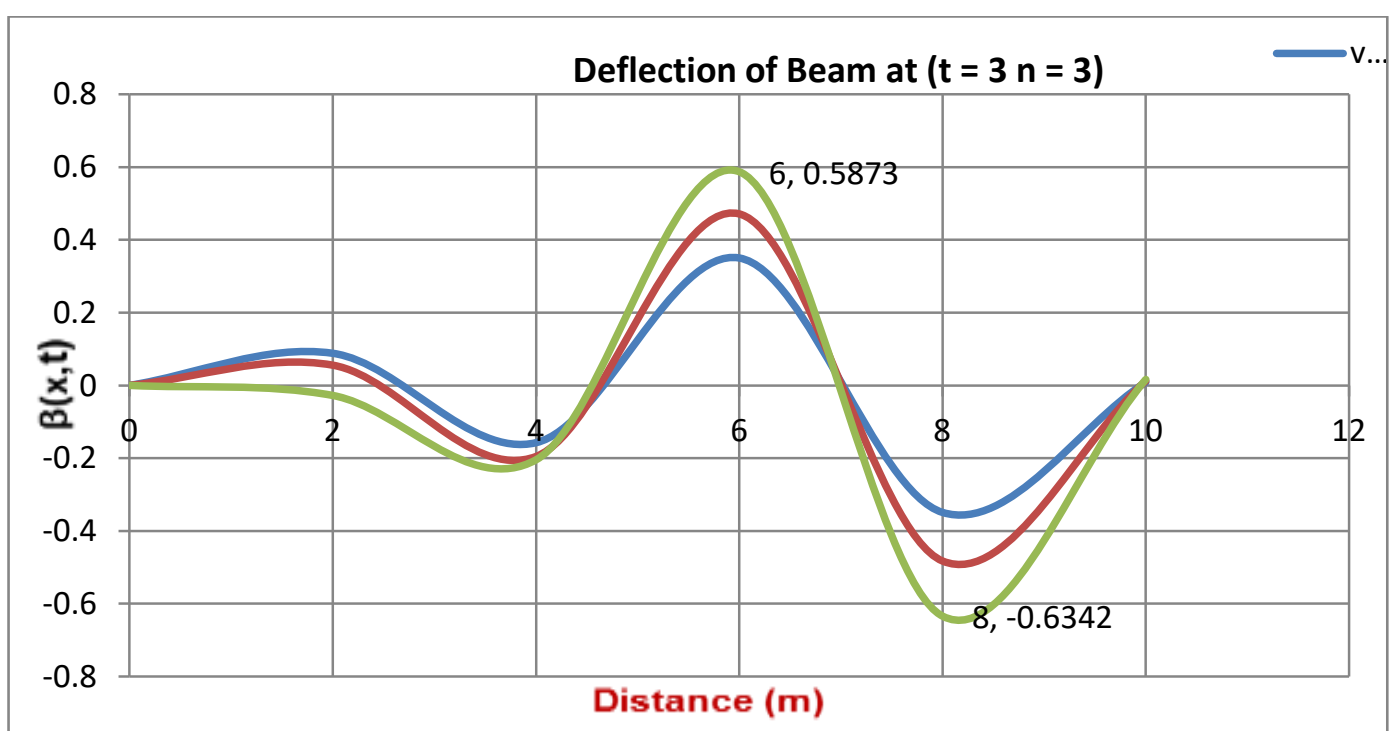

Figure 6-Graph of deflection at $t=3 s(N P)$ and $n=3$

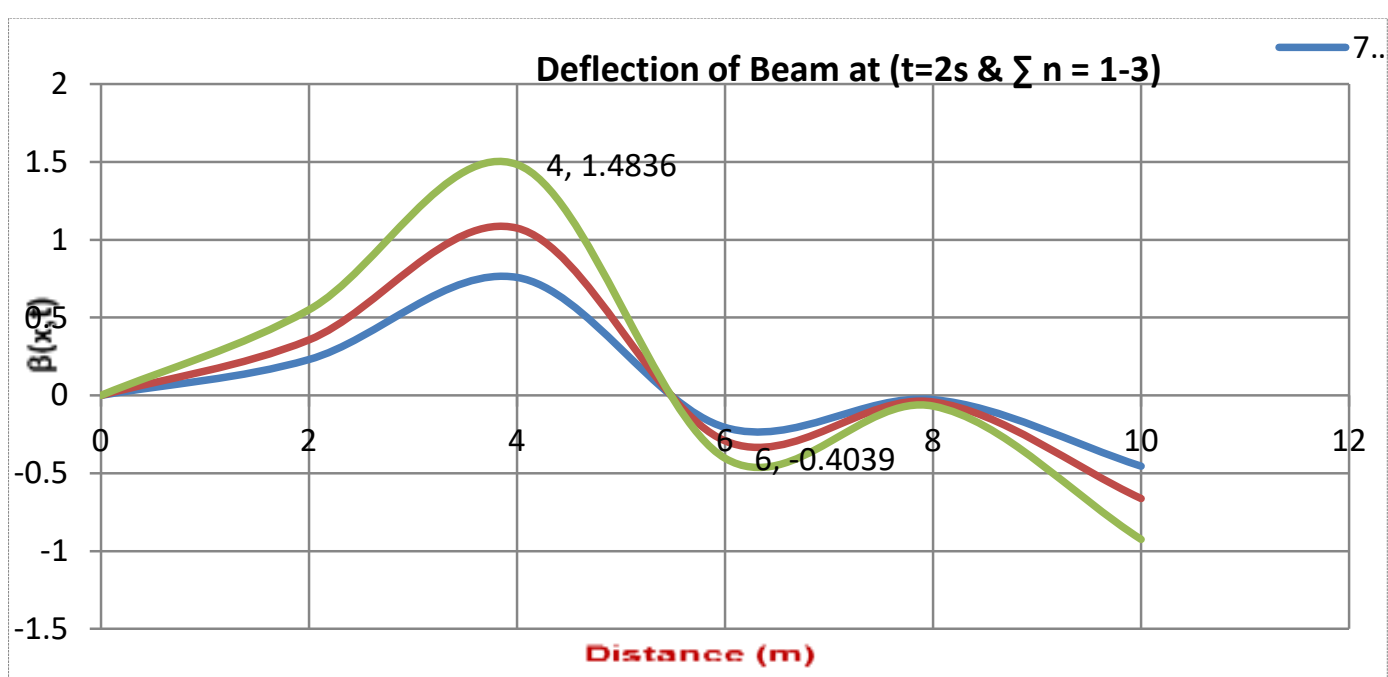

Figure 7-Graph of deflection at $\mathrm{t}=2 \mathrm{~s}(\mathrm{NP})$ and $\sum_{n=1}^{3}$. 


\section{Conclusions}

This study demonstrates the vibration analysis of Euler-Bernoulli beam resting on a Winkler foundation subjected to a concentrated distributed moving load. Fourier transform method was employed to find the exact solution of the governing partial differential equation of the beam of length $\mathrm{L}$ on both cases. Modal analysis was also applied to convert the P.D.E to O.D.E. in equation (1.0). Numerical calculations were performed to analyse the deflection and bending moment responses of the beam on a Winkler foundation subjected to a concentrated load with different velocity_and a free load with mass of the beam.

The results show that the increment in velocity leads to increase in both the displacement and bending moments of the beam. It further shows that the deflection and the bending moment decreases as the number of the contributed mode increases.

Finally, it can be concluded that the deflection of the beam increases as the velocity of the load increases. Bending moment also increases in response to the increase in velocity and time.

\section{REFERENCES}

1. Rajesh K. and MeeraSahebKoppanati 2016. Linear free vibration analysis of taperedTimoshenko beams using coupled displacement field Method.

2. Abrate S. 1995. Vibration of non-uniform rods and beams. Journal of Sound and Vibration. 185(4): 703-716.

3. Byoung Koo Lee, et al 2002. Free vibrations of tapered Beams with general boundary condition. Journal of Civil Engineering, 6(3): 283-288.

4. Arıkoğlu A, Özkol İ. 2005. Solution of boundary value problems for integro-differential equations by using differential transform method. Journal of Applied Mathematics and Computation, 168: $1145-1158$.

5. Ayaz F. 2003. On the two-dimensional differential transform method. Journal of Applied Mathematics and Computation. 143: 361-374.

6. Chen C. N. 2002. DQEM vibration analyses of non-prismatic shear deformable beams resting on elastic foundations. Journal of Sound and Vibration, 255(5): 989-999.

7. Özdemir Ö. and Kaya M. O. 2006. Flap-wise bending vibration analysis of a rotating tapered cantilever Bernoulli-Euler beam by differential transform method. Journal of Sound and Vibration, 289: 413-420.

8. Mahammad Amin R. and Al Amin R. 2013. 'Analysis of vibration of a pipeline supported on an elastic soil using differential transforms method'. American Journal of Mechanical Engineering 1. 4: 96-102.

9. Chen W. Q, Lü C. F. And Bian Z. G. 2004. A mixed method for bending and free vibration of beams resting on a Pasternak elastic foundation. Journal of Applied Mathematical Modelling, 28: 877-890.

10. Coşkun İ. 2003. The response of a finite beam on a tensionless Pasternak foundation subjected to a harmonic load. European Journal of Mechanics and Solids, 22: 151-161.

11. De Rosa M. A. 1995. Free vibration of Timoshenko beams on two-parameter elastic foundation. Journal of Computers and Structures, 57(1): 151-156.

12. Elfelsoufi Z. And Azrar L. 2005. Buckling, flutter and vibration analyses of beams by integral equation formulations. Journal of Computers and Structures, 83: 2632-2649.

13. Firouz-Abadi R. D, et al 2007. An asymptotic solution to transverse free vibrations of variablesection beams. Journal of Sound and Vibration, 304: 530-540.

14. Fryba L. 1972. Vibration of Solids and Structures under Moving Loads. Groningen, Noordhoff International.

15. Idowu, A. S., Are E. B, Joseph, K. M. and Daniel, S. K. 2013. Dynamic effects of viscous damping on isotropic rectangular plates resting on Pasternak foundation subjected to moving loads, International journal of Mathematics and Statistics Studies. 1(2): 12-19.

16. zabelaZamorska 2010. Free Transverse vibrations of non-uniform beams. Scientific Research of the Institute of Mathematics and Computer Science, 9(2): 244-250. 
17. Kolousek V. 1967, 1956, 1961. Dynamics of Civil Engineering Structures. Part I — General Problems, second edition; Part II - Continuous Beams and Frame Systems, second edition; Part III - Selected Topics (in Czech.) SNTL, Prague.

18. Lee H.P. 1996. 'Dynamic response of a beam with a moving mass'. Journal of Sound and Vibration, 191: 298-294.

19. Mahmoud A. A., et al 2013. Free vibrations of uniform and non-uniform Euler beam using differential transformation method. Asian Journal of Mathematics and Applications, 13: 1-16.

20. Mehmet CemEce, et al. 2007. Vibration of a variable cross-section beam. Journal of Mechanics Research Communications, 34: 78-84

21. Michaltsos G. T. and Kounadis A. N. 2001. The Effects of Centripetal and Coriolis Forces on the Dynamic Response of Light (steel)Bridges under Moving Loads, journal of vibration and control,7(3): 315-326.

22. Mohamed HussienTaha, Samir Abohadima 2008. Mathematical model for vibrations of nonuniform flexural beams. Journal of Engineering Mechanics, 15(1): 3-11.

23. MügeBalkaya, Metin O. Kaya, Ahmet Sağlamer 2008. Analysis of the vibration of an elastic bean supported on Elastic soil using differential Transform Method'. Archive of Applied Mathematics, 79(2): 135-146.

24. Reza Attarnejad and Ahmad Shabba 2008. 'application of Differential Transform method in Free Vibration Analysis of rotating Non-Prismatic Beam. World Applied Science Journal. 5(4): 441448.

25. Rossi R. E. and Laura P. A. A. 1993. Numerical experiments on vibrating, linearly tapered Timoshenko beams. Journal of Sound and Vibration, 168(1): 179-183.

26. SaeidBashash, Amin Salehi-Khojin, Nader Jalili 2008. Forced vibration of flexible Euler-Bernoulli Beams with Geometrical Discontinuities. American Control conference 4029-4034

27. Stokes G. G. 1983. Discussions of a differential equation relating to the breaking of railway bridges. Mathematical and Physical Papers, 2: 178-220.

28. Ting E. C, Genin J. and Ginsberg J. H. 1974. A general algorithm for moving mass problems. Journal of Sound and Vibration, 33: 49-58.

29. Usman M.A. 2003. Dynamic Response of a Bernoulli Beam on Winkler Foundation under the action of partially distributed load. Nigerian Journal of Mathematics and Application, 16(1): 128147.

30. Willis R. et al. 1994. Preliminary essay to the appendix B. Experiments for determining the effects produced by causing weights to travel over bars with different velocities. In: Grey G. et al.: Report of the Commissioners Appointed to inquire into the application of iron to railway structures. 